\title{
Early Donepezil Monotherapy or Combination With Metoprolol Significantly Prevents Subsequent Chronic Heart Failure in Rats With Reperfused Myocardial Infarction
}

Meihua Li ( $\nabla$ limeihua@ncvc.go.p )

National Cerebral and Cardiovascular Center https://orcid.org/0000-0003-1184-5482

Can Zheng

National Cerebral and Cardiovascular Center

Kawada Toru

National Cerebral and Cardiovascular Center

Kazunori Uemura

National Cerebral and Cardiovascular Center: Kokuritsu Junkankibyo Kenkyu Center

Inagaki Masashi

National Cerebral and Cardiovascular Center

Takuya Nishikawa

National Cerebral and Cardiovascular Center

Keita Saku

National Cerebral and Cardiovascular Center

Masaru Sugimachi

National Cerebral and Cardiovascular Center

Routine Article

Keywords: chronic heart failure, donepezil, metoprolol, myocardial salvage, infarct size, reperfused myocardial infarction

Posted Date: February 5th, 2021

DOl: https://doi.org/10.21203/rs.3.rs-175205/v1

License: (c) (1) This work is licensed under a Creative Commons Attribution 4.0 International License. Read Full License 


\section{Abstract}

\section{Purpose}

Acute myocardial infarction (MI) remains a leading cause of chronic heart failure, and $\mathrm{MI}$ size is a strong predictor of prognosis. Clinical guidelines recommend that oral $\beta$-blockers be initiated early after reperfused $\mathrm{MI}(\mathrm{RMI})$. Here, we compared the effects of donepezil, metoprolol, and their combination on infarct size and progression of cardiac remodeling in rats with RMI.

\section{Methods}

RMI $(n=103)$ was induced in 8-week-old male rats by occluding the left coronary artery (30 min), followed by reperfusion. The animals were randomly assigned to untreated (UT, $n=16$ ), donepezil-treated (DT, $5 \mathrm{mg} / \mathrm{kg} \cdot$ day, $n=21$ ), metoprolol-treated (MT, $70 \mathrm{mg} / \mathrm{kg} \cdot$ day, $n=23$ ), and combination of donepezil and metoprolol (DMT, $n=18)$ groups.

\section{Results}

On day 8 after surgery, rats in the DT and DMT groups exhibited significantly improved myocardial salvage, owing to the prevention of apoptosis and suppression of macrophage infiltration, compared with rats in the UT group. After the 10-week treatment, the rats in the DT and DMT groups exhibited decreased heart rate, reduced MI size, attenuated cardiac hypertrophy and cardiac dysfunction, and decreased plasma levels of brain natriuretic peptide, norepinephrine, and high-sensitivity C-reactive protein. However, no cardioprotective effects were observed among rats in the MT group.

\section{Conclusions}

Donepezil monotherapy or in combination with metoprolol significantly improved myocardial salvage, reduced $\mathrm{MI}$ size, limited the progression of cardiac remodeling, and prevented subsequent chronic heart failure in rats with RMI. Thus, donepezil monotherapy or combination with metoprolol may be applied as pharmacotherapy post-RMI.

\section{Introduction}

Timely reperfusion via primary percutaneous coronary intervention $(\mathrm{PCl})$ is the best therapeutic strategy for patients with acute myocardial infarction (AMI), and its widespread implementation has significantly reduced patient mortality [1]. Nevertheless, many individuals with AMI develop cardiac remodeling and chronic heart failure (CHF) because of the loss of viable myocardium, even after reperfusion therapy [2$4[2-4]$. Therefore, it is necessary to salvage more myocardium during the management of AMI to prevent subsequent $\mathrm{CHF}$.

Sympathetic overactivity is involved in the pathology of CHF. Clinical practice guidelines recommend early $(<24 \mathrm{~h})$ oral administration of $\beta$-blockers, such as metoprolol [5], after coronary reperfusion in 
patients with AMI. However, oral metoprolol administration, which is initiated early post-reperfusion, does not improve myocardial salvage in patients with AMI [6]. Several experimental studies and clinical trials have reported controversial effects of early intravenous metoprolol administration before coronary reperfusion on myocardial salvage, $\mathrm{Ml}$ size, and cardiac remodeling, and improvements in endpoints of efficacy were not confirmed in a larger population [7-10]. Decreased parasympathetic activity is another independent risk factor following AMI [11, 12]; however, few studies have investigated treatment alternatives to combat parasympathetic dysfunction.

Parasympathetic activation via electrical vagal nerve stimulation prevents cardiac remodeling and improves the long-term survival of rats with $\mathrm{CHF}$ [13]. Moreover, early vagal nerve stimulation attenuates cardiac remodeling after reperfused $\mathrm{MI}$ (RMI) in rabbits [14]. Recently, we proposed a novel pharmacotherapeutic approach using donepezil, an acetylcholinesterase inhibitor, to improve parasympathetic function. Oral administration of donepezil, as monotherapy or in combination with losartan, prevents the progression of cardiac remodeling and improves the long-term prognosis in rats with $\mathrm{CHF}$ with permanent $\mathrm{MI}[15,16]$. Thus, adding donepezil to conventional $\beta$-blocker therapy may improve the autonomic balance and prevent CHF.

Here, we examined whether early administration of donepezil, metoprolol, or their combination in a rat model of RMI affected myocardial salvage, infarct size, cardiac remodeling, cardiac dysfunction, and the development of CHF. Our findings provide a rationale for early administration of donepezil as an adjunct therapy to $\mathrm{PCl}$, thereby improving the long-term prognosis of patients with AMI.

\section{Methods}

Animals were housed and studied in accordance with Directive 2010/63/EU of the European Parliament and the Guiding Principles for the Care and Use of Animals in the Field of Physiological Science. The investigation conformed to the NIH Guide for the Care and Use of Laboratory Animals (NIH Publication No. 85 - 23, revised 1985). All protocols were reviewed and approved by the Animal Subject Committee of the National Cerebral and Cardiovascular Center (Osaka, Japan). The experiment comprised two studies: myocardial salvage and cardiac remodeling. During experiments, the animals were anesthetized with halothane ( $3 \%$ at induction, $1.2 \%$ during surgery, and $0.6 \%$ during data recording), and the body temperature was maintained at $37^{\circ} \mathrm{C}$. For details, see the Methods section of the online Supplementary Material.

\section{RMI model}

Eight-week-old male Sprague-Dawley rats (SLC, Inc., Hamamatsu, Japan) were used $(n=141)$. After inducing anaesthesia, the heart was exposed, and the pericardium was opened via a left lateral thoracotomy at the second intercostal space. A 5- 0 prolene suture was placed around the left ventricular proximal coronary artery (LCA) to create a reversible snare. All rats underwent a total of 30 minutes of 
LCA occlusion, followed by reperfusion and closed the chest. The loosened snare was kept in the chest for identifying the ischaemic regions later.

\section{Experimental protocol}

Shortly after induction of RMI, 23 rats were used for the myocardial salvage study, and 80 rats were used for the cardiac remodeling study (Fig. 1A). All drug treatments were initiated $1 \mathrm{~h}$ after recovery from anaesthesia. Rats with RMI were randomly divided into the untreated (UT), donepezil-treated (DT), metoprolol-treated (MT), and donepezil plus metoprolol-treated (DMT) groups. For the DT and DMT groups, donepezil was dissolved in drinking water to provide a dose of $5 \mathrm{mg} / \mathrm{kg} \cdot$ day. For the MT and DMT groups, metoprolol was dissolved in drinking water at a dose of $70 \mathrm{mg} / \mathrm{kg} \cdot d a y$. These dosages were selected as previously described $[17,15]$.

\section{Myocardial salvage study}

After 1 week of treatment, rats with RMI (Fig. 1B) were analysed as described below.

\section{Determination of neutrophil infiltration}

The middle of the short-axis portion of the left ventricle (LV) was embedded in paraffin, sectioned transversely to 4- $\mu \mathrm{m}$ thickness, and stained with Masson's trichrome. Neutrophils were counted using a light microscope (BZ-9000; KEYENCE, Osaka Japan) coupled to an image-analysis software at 40x magnification in six fields, which were randomly selected from the ischaemic/reperfusion (I/R) region per rat.

\section{Analysis of myocardial apoptosis}

Myocardial apoptosis in the I/R region was analysed. Apoptotic cells were detected using terminal deoxynucleotidyl transferase dUTP nick end labelling (TUNEL) staining [18]. Biventricular sections were deparaffinized and incubated with the TUNEL reagent and rabbit anti-connexin 43 antibody, followed by incubation with Alexa Fluor 633-conjugated goat anti-rabbit IgG antibody. The fluorescence of Alexa Fluor 488 (TUNEL), Alexa Fluor 633 (connexin 43), and Alexa Fluor 350 (nuclei) was observed under a fluorescence microscope. Apoptotic myocardial cells were counted at 20x magnification. Data obtained from six fields were averaged and expressed as the number of apoptotic cells.

\section{Examination for macrophage infiltration}

Myocardial salvage associated with myocardial inflammation in the I/R region, which was assessed by observing for macrophage infiltration. Tissue sections were prepared to examine macrophage infiltration and incubated with mouse anti-CD68 and rabbit anti-human von Willebrand factor (vWF) polyclonal antibodies. Sections were then incubated with Alexa Fluor 488-conjugated goat anti-mouse IgG and Alexa Fluor 633-conjugated goat anti-rabbit IgG antibodies. The fluorescence of Alexa Fluor 488 (CD68), Alexa Fluor 633 (vWF), and Alexa Fluor 350 (nuclei) was observed using a fluorescence microscope at 40x magnification. To distinguish between classically activated pro-inflammatory type 1 macrophages (M1) 
and alternatively activated anti-inflammatory type 2 macrophages (M2), the following were stained together: (1) CD68, CD80, and DAPI for M1; or (2) CD68, CD163, and DAPI for M2 [19]. Data obtained from six fields were averaged and expressed as the percentage area of macrophage infiltration.

\section{Cardiac remodeling study}

\section{Telemetric measurements of blood pressure (BP) and heart rate (HR)}

Rats were anesthetized and ventilated through an endotracheal cannula during the implantation procedure. Rats were implanted with BP transmitters (TA11PA-C40; DSI, St. Paul, MN, USA) for real-time monitoring of the hemodynamics of conscious rats. BP transmitter catheters were inserted into the abdominal aorta. Data on BP and HR of freely moving animals were recorded continuously. The recording was sampled at $500 \mathrm{~Hz}$. One week after implantation of BP transmitters, RMI was induced, and 80 surviving rats were randomly assigned to the UT, DT, MT, and DMT groups. After 10 weeks of treatment, the surviving rats (Fig. 1C) were analysed.

\section{Hemodynamic measurements and assessment of cardiac remodeling under anaesthesia}

Ten weeks after RMI, the hemodynamics and heart weights of rats with CHF were determined. LV and arterial pressures were measured with a 2-Fr catheter-tip micromanometer (SPC-320; Millar Instruments), and aortic flow was measured with a flow probe (Transonic Systems Inc.; T-206 flow probe \#2.5 ss66). Right atrial pressure (RAP) was measured using a fluid-filled pressure sensor. All signals were digitized at $500 \mathrm{~Hz}$ for $5 \mathrm{~min}$. After hemodynamic measurements, $2 \mathrm{~mL}$ blood was sampled and transferred into an EDTA.2Na tube. Neurohumoral and cytokine assays were performed with the plasma, which was obtained after centrifugation of the blood $(3000 \cdot \mathrm{g}, 20 \mathrm{~min})$ at $4^{\circ} \mathrm{C}$.

\section{Neurohumoral and cytokine assays}

Plasma concentrations of catecholamines were measured using high-performance liquid chromatography with electrochemical detection after alumina adsorption [15]. Plasma levels of brain natriuretic peptide (BNP) and high-sensitivity C-reactive protein (hs-CRP) were determined using enzymelinked immunosorbent assay kits, per the manufacturer's instructions.

\section{Histological assessments of risk area, infarct size, cardiac fibrosis, and myocyte cross-sectional area}

Rats were euthanized with an overdose of pentobarbital sodium (100 mg/kg, i.v.) after hemodynamic measurements and blood sampling during anaesthesia. Hearts were harvested and mounted on a modified $[19,18]$ Langendorff apparatus. The perfusate was infused with Pigment Blue Ink (PLATINUM CO., LTD., Japan) to identify the ischaemic regions. Once the dye had stained the non-ischaemic regions, biventricular weights were determined, and the hearts were sectioned. Three portions (1-mm thick) from 
the apex to the base were selected and embedded in paraffin. Sections (4- $\mu \mathrm{m}$ thick) were cut and stained with Masson's trichrome. Histological images were obtained with a microscope. The area at risk was calculated as the percentage of the unstained I/R region relative to the whole LV area, which was averaged for the three portions. The infarcted area was calculated as the percentage of the area of scar tissues relative to the whole LV area, which was averaged for the three portions. MI size was defined as the percentage of the infarcted area relative to the area at risk. The extent of cardiac fibrosis was evaluated using a light microscope at 20x magnification, and myocyte cross-sectional area (papillary cardiomyocytes) was evaluated at $40 \times$ magnification. The area of cardiac fibrosis in each heart was calculated as the ratio of the blue area to the total tissue area in eight fields of the non-infarcted area.

\section{Immunohistochemical examination and analysis of microvessel density}

Biventricular sections were pretreated as described in the myocardial salvage study. Sections were incubated with a rabbit anti-human vWF polyclonal antibody and then with Alexa Fluor 633-conjugated goat anti-rabbit IgG antibody for analysis of microvessel density. Capillary vessels in the peri-infarct area and septum were counted as fluorescent regions using Alexa Fluor 633 (vWF) and a laser-scanning microscope at 20x magnification. Data obtained from 2-5 fields were averaged and expressed as the number of capillary vessels.

\section{Statistical analysis}

All statistical analyses were performed using GraphPad Prism 7 (GraphPad Software, San Diego, CA, USA). All data were expressed as means \pm standard errors of the means (SEMs). Long-term-recorded data on HR and mean BP (MBP) before and during treatment within each group were compared using repeated measures one-way analysis of variance (ANOVA), followed by Dunnett's test. For data obtained from the hemodynamic and remodeling study, differences between groups were tested using one-way ANOVA, followed by Dunnett's multiple comparisons test. Data on the myocardial salvage study, cardiac fibrosis, $\mathrm{MI}$ size, microvessel density, neurohumoral, and cytokine assays were analysed using nonparametric Kruskal-Wallis tests, followed by Dunn's tests for all pairwise comparisons. Differences were considered significant at the level of $P<0.05$.

\section{Results}

\section{Immune cell infiltration into cardiac cells and myocardial apoptosis}

Figure 2 shows representative images of Masson's trichrome (Fig. 2A, B, S1) and TUNEL-Cox43 (Fig. 2D) staining of hearts on day 8 after RMI. Considerable neutrophil infiltration into the myocardium of RMI tissues was observed in the UT and MT groups, with significant reductions in the DT and DMT groups (Fig. 2C). Apoptotic cell counts were significantly lower in the DT and DMT groups than in the UT and MT groups (Fig. 2E), with strong connexin43 signals in the DT and DMT groups. 
Immunohistochemical staining of $\mathrm{CD} 68^{+}$cells (all macrophages), $\mathrm{CD} 68^{+}-\mathrm{vWF}{ }^{+}$cells (microvessels), $\mathrm{CD}^{2} 8^{+}-\mathrm{CD} 80^{+}(\mathrm{M} 1)$ cells, and $\mathrm{CD} 68^{+}-\mathrm{CD} 163^{+}$(M2) cells in RMI tissues revealed decreased total macrophage infiltration, decreased $M 1$ cells, and increased $M 2$ cells and microvessels in the I/R region in the DT and DMT groups, compared with that in the UT and MT groups (Fig. 3A-H).

\section{Telemetric hemodynamic measurements in conscious rats with RMI-induced CHF}

In the cardiac remodeling study, the weekly average HR in the UT group increased to $413 \mathrm{bpm}$ at week 1 during pretreatment and then decreased (Fig. 4A). Compared with the UT group, the weekly average HR in the other groups was significantly reduced. This reduced HR was prominent in the DMT group. The difference in HR between the DMT and UT groups was significant and approximately $40 \mathrm{bpm}$ at week 4 of treatment. Figure 4B shows changes in daily average HR during the first 7 days. The HR increased, reached a maximum (425 bpm) on day 3 , and then gradually decreased in the UT group. The difference in HR between the DMT and UT groups was significant and approximately $60 \mathrm{bpm}$ on day 7. Similarly, the HR in the DT and MT groups was decreased significantly compared with that in the UT group. The weekly MBP in the DT, MT, and DMP groups increased from that at week 0 , but did not significantly change in the UT group (Table S1). The differences in MBP reached approximately $10 \mathrm{mmHg}$ at the end of week 4 .

\section{Hemodynamics under anaesthesia, cardiac remodeling, and cardiac function}

The parameters of hemodynamics, cardiac remodeling, and cardiac function after 10 weeks of treatment are shown in Table 1. Body weights of the rats in the DT, MT, and DMT groups were higher than those in the UT group. Rats in the DT and DMT groups had lower heart weights, higher cardiac index (CI), lower LV end-diastolic pressure (LVEDP), higher maximum dp/dt LV pressure ( $\left.L V \mathrm{dp} / \mathrm{dt}_{\max }\right)$, and minimum $\mathrm{dp} / \mathrm{dt}$ $\mathrm{LV}$ pressure $\left(\mathrm{LV} \mathrm{dp} / \mathrm{dt}_{\min }\right)$ than the rats in the UT group. 
Table 1

Hemodynamic, cardiac remodeling, and plasma neurohumoral and inflammatory parameters in rats with RMI-induced chronic heart failure (CHF) after 10 weeks of treatment

\begin{tabular}{|c|c|c|c|c|}
\hline & $\begin{array}{l}\text { UT group } \\
(n=12)\end{array}$ & $\begin{array}{l}\text { DT group } \\
(n=15)\end{array}$ & $\begin{array}{l}\text { MT group } \\
(n=18)\end{array}$ & $\begin{array}{l}\text { DMT group } \\
(n=14)\end{array}$ \\
\hline BW, g & $495 \pm 7$ & $523 \pm 7$ & $525 \pm 10$ & $545 \pm 9 * *$ \\
\hline $\mathrm{HW}, \mathrm{g} / \mathrm{kg}$ & $2.73 \pm 0.13$ & $2.39 \pm 0.05^{\star}+\dagger$ & $2.73 \pm 0.06$ & $2.41 \pm 0.03^{*}+$ \\
\hline Infarcted area, \% & $20.1 \pm 1.2$ & $12.5 \pm 1.5^{\star \star}+\dagger$ & $21.1 \pm 1.1$ & $14.3 \pm 1.7^{\star * 十+}$ \\
\hline MBP, mmHg & $96 \pm 3$ & $98 \pm 3$ & $96 \pm 3$ & $107 \pm 3$ \\
\hline $\mathrm{HR}$, bpm & $302 \pm 7$ & $312 \pm 6$ & $304 \pm 4$ & $317 \pm 7$ \\
\hline $\mathrm{Cl}, \mathrm{mL} / \mathrm{min} / \mathrm{kg}$ & $98 \pm 6$ & $120 \pm 4^{\star}+\dagger$ & $91 \pm 4$ & $120 \pm 4^{\star}+\dagger$ \\
\hline $\mathrm{LV} \mathrm{dp} / \mathrm{dt}_{\max }, \mathrm{mmHg} / \mathrm{s}$ & $4318 \pm 127$ & $4853 \pm 173^{\star}$ & $4387 \pm 180$ & $4950 \pm 120 *+$ \\
\hline 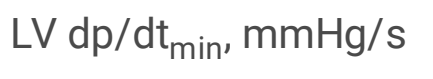 & $3493 \pm 147$ & $4022 \pm 154^{*}+$ & $3511 \pm 166$ & $4277 \pm 113^{\star \star \star 十 † ~}$ \\
\hline LVEDP, $\mathrm{mmHg}$ & $23 \pm 3$ & $14 \pm 2^{\star}$ & $21 \pm 2$ & $15 \pm 1 *$ \\
\hline RAP, $\mathrm{mmHg}$ & $2.9 \pm 0.3$ & $3.1 \pm 0.4$ & $3.4 \pm 0.3$ & $2.6 \pm 0.5$ \\
\hline $\begin{array}{l}\text { Plasma: } \\
\text { NE, pg/mL }\end{array}$ & $392 \pm 96$ & $155 \pm 27^{*}$ & $282 \pm 58$ & $111 \pm 18^{\star \star}+$ \\
\hline Epi, pg/mL & $430 \pm 85$ & $112 \pm 55^{\star \star 十 †}$ & $381 \pm 92$ & $119 \pm 22^{\star \star}+$ \\
\hline BNP, pg/mL & $553 \pm 42$ & $370 \pm 16^{\star \star \star 十 †}$ & $482 \pm 20$ & $417 \pm 8^{\star \star}$ \\
\hline hs-CRP, ng/mL & $825 \pm 67$ & $262 \pm 61^{\star \star}$ & $415 \pm 38 *$ & $201 \pm 12^{\star *+†}$ \\
\hline \multicolumn{5}{|c|}{ 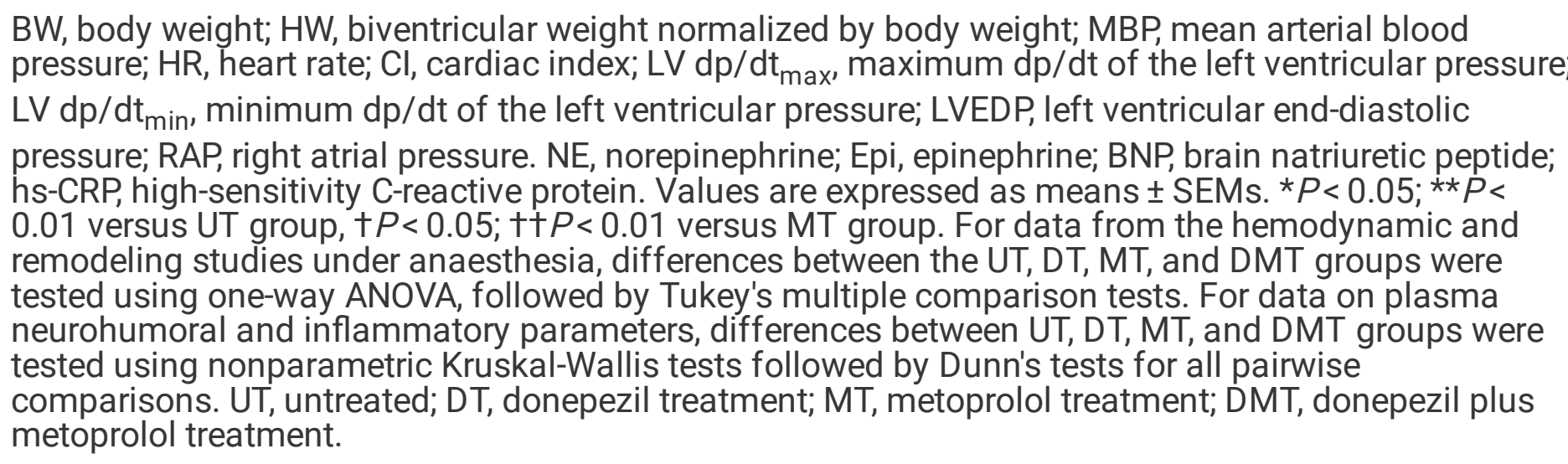 } \\
\hline
\end{tabular}

\section{Histological assessments of risk area, Ml size, cardiac fibrosis, and myocyte cross-sectional area}


Among rats in the DT and DMT groups, histological analyses revealed the association of preserved cardiac function with histological changes (Fig. 5A-C, S2). Rats in the DT and DMT groups showed a significantly reduced MI size compared with that in the UT group. Similarly, among the rats in the DT and DMT groups, cardiac fibrosis was significantly suppressed, and cardiac myocyte hypertrophy was markedly attenuated as a direct consequence of the reduced myocyte cross-sectional area (Fig. 5D-G). These beneficial effects were not observed among the rats in the MT group.

\section{Analysis of microvessel density}

Immunohistochemical analysis of vWF in the peri-infarct area and septum revealed that neovascularization in the DT and DMT groups was more pronounced than that in the UT group (Fig. 5H). Quantitative analysis confirmed a significantly higher capillary density in the peri-infarct and septum regions in the DT and DMT groups than in the UT group. Metoprolol monotherapy did not promote angiogenesis in RMl-induced rats with CHF (Fig. 5l, J).

\section{Neurohumoral and cytokine levels}

Table 1 shows the plasma levels of neurohumoral and inflammatory markers after 10 weeks of treatment. Rats in the DT and DMT groups showed lower plasma levels of norepinephrine, epinephrine, and BNP and reduced hs-CRP levels compared with rats in the UT group. Metoprolol monotherapy reduced plasma hs-CRP levels compared with those in the UT group.

\section{Discussion}

We investigated the effects of early administration of donepezil, metoprolol, and their combination on cardiac function in a rat model of RMI. We showed that early administration of donepezil (with or without metoprolol) facilitated myocardial salvage, prevented HR increase and preserved MBP, reduced MI size, prevented the progression of cardiac remodeling and dysfunction, suppressed catecholamine and BNP plasma levels, promoted angiogenesis, and suppressed inflammation in the heart. Thus, early administration of donepezil (with or without metoprolol) prevented structural changes in the heart after RMI, probably via activation of the vagal nerve. Local anti-inflammatory effects on the heart early in treatment may contribute to myocardial salvage. Early administration of donepezil may be effective for post-RMI therapy.

\section{Early donepezil treatment (with or without metoprolol) affected myocardial salvage post-RMI via an anti- inflammatory mechanism}

Donepezil (with or without metoprolol), initiated early post-RMI, preserved myocardial salvage by suppressing neutrophil infiltration and myocardial apoptosis in the I/R region, 1 week after RMI. These treatments also attenuated total macrophage infiltration and suppressed local inflammation by reducing 
the production of pro-inflammatory M1 cells and promoting the production of anti-inflammatory M2 cells. The M1/M2 ratio was reduced in the I/R region. Suppression of local inflammation reduced infarct size, limited cardiac remodeling, preserved cardiac function and prevented subsequent progression to $\mathrm{CHF} 10$ weeks post-RMI. When uncontrolled, these immune responses, which are related to the structural changes after RMI, induce cardiac remodeling, leading to CHF. Specifically, MI size is a strong predictor of cardiac remodeling, dysfunction, and cardiovascular events, which subsequently progress to CHF [20]. Furthermore, myocardial salvage is associated with the effects of myocardial inflammation, including myocardial apoptosis and macrophage/neutrophil infiltration in the I/R region, early post-RMI [6]. These observations are consistent with our previous reports showing anti-inflammatory effects of donepezil in rats with $\mathrm{CHF}$ and permanent MI $[15,21]$.

\section{Early donepezil, metoprolol, and combined donepezil-metoprolol therapy reduced HR and affected MBP preservation in RMl-induced CHF}

We used a telemetric system to monitor arterial MBP and HR in conscious, unstressed rats. Donepezil treatment (from week 2), metoprolol treatment, or their combination (from week 1) reduced the weekly average HR compared with that in the UT group. In particular, combined treatment significantly decreased the immediate increase in HR from day 2 post-RMI, and metoprolol treatment decreased the increase in HR from day 3 post-RMI. Furthermore, weekly HR in the DMT group nearly reached that of healthy animals at week 4 post-RMI [13]. The observed reduction in HR may have resulted from a decreased sympathetic drive and increased efferent discharges of the parasympathetic system [12]. Although we did not directly evaluate sympathetic nerve activity, low plasma levels of norepinephrine and epinephrine in the DT and DMT groups may reflect decreased central sympathetic outflow [22]. The bradycardia observed in the DT and DMT groups may play important roles in preventing cardiac dysfunction. Additionally, the effects of bradycardia were observed in the MT group. Metoprolol is a $\beta$-blocker, which induces bradycardia, and is important for the treatment of patients with CHF. However, because the sinus node and cardiac myocytes express $\beta$-adrenergic receptors, $\beta$-blockers decrease HR and suppress myocardial contractility. Hence, considering the negative inotropic effect, $\beta$-blockers may not be suitable for patients with poor hemodynamics or decompensation with pre-existing myocardial dysfunction because the maintenance of cardiac output in such patients partly depends on an increased sympathetic drive. Hence, although metoprolol treatment led to a long-term decrease in HR, it did not significantly

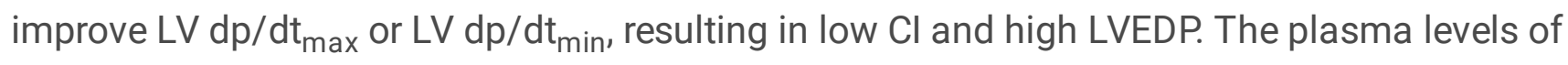
catecholamines were also not significantly affected in the MT group compared with the levels in the UT group. Although metoprolol treatment blocks peripheral sympathetic effects, it did not decrease the central sympathetic outflow in CHF. Vasoconstriction could occur via unblocked a-adrenergic receptors, which may have contributed to the increased MBP in the MT and DMT groups. However, donepezil treatment also increased MBP over the baseline (week 0 ) values. Because the vagus nerve does not directly affect ventricular contractility, increases synaptic acetylcholine levels, and reduces HR, the negative effects on the pumping function of the heart are lower with donepezil than with metoprolol. Prevention of cardiac dysfunction may also have contributed to the maintenance of MBP, resulting in 
arterial baroreflex-mediated inhibition of sympathetic nerve activity and HR reduction [23]. A prolonged cardiac cycle is beneficial for the enhancement and maintenance of cardiac function by decreasing myocardial oxygen consumption, increasing coronary blood flow, and increasing ventricular filling time [24]. Since acetylcholine antagonizes the effects of $\beta$-adrenergic stimulation [25], donepezil may be either an alternative to $\beta$-blockers or adding to the conventional $\beta$-blockers therapy in patients with CHF postRMI.

\section{Early donepezil (with or without metoprolol) affected cardiac remodeling and dysfunction}

Infarct size is a strong predictor of cardiovascular events. We showed that donepezil treatment, as monotherapy or in combination with metoprolol, reduced infarct size, prevented cardiac fibrosis and cardiomyocyte hypertrophy, and increased microvessel density. These treatments prevented the progression of cardiac remodeling and dysfunction compared with that in untreated rats. Plasma catecholamine levels were also significantly reduced in the DT and DMT groups, consistent with our previous studies $[15,16]$. Furthermore, plasma BNP levels in the DT and DMT groups were significantly reduced compared with those in the UT group, which may have resulted from the augmented vagal tone and decreased central sympathetic outflow [22]. These observations showed that early administration of donepezil (with or without metoprolol) after RMI is beneficial mainly via local anti-inflammatory effects and prevention of myocardial apoptosis, showing myocardial salvage in the acute phase. Metoprolol monotherapy did not exert these beneficial effects, but reduced hs-CRP levels and HR and preserved MBP. The reduced hs-CRP levels in the MT group may indicate an indirect reduction in the inflammatory response of the tissues, which is associated with reduced shear stress due to long-term reduction of HR.

\section{Probable mechanisms of the effects of early administration of donepezil (with or without metoprolol)}

In the DT and DMT groups, suppression of local inflammation 1 week after RMI may be associated with decreased plasma hs-CRP levels and increased microvessel density in the peri-infarct and remote regions after 10 weeks of treatment. This leads to more pronounced myocardial salvage than in the UT group by preventing enlargement of MI size and suppressing cardiac fibrosis and cardiomyocyte hypertrophy. Although $\mathrm{PCl}$ entails second-generation drug-eluting stents and reduces post-AMI care, the incidence of post-AMI CHF remains high; every $5 \%$ increase in infarct size is associated with a $20 \%$ increase in 1 -year hospitalisation for CHF and 1-year mortality [26-28]. Here, we showed that infarct size was decreased by $35 \%$ and $31 \%$ in the DT and DMT groups, respectively, compared with that in the untreated group. Metoprolol treatment at 2 weeks after permanent MI improved cardiac function [17]; however, in this study, early metoprolol monotherapy did not exert beneficial effects, excluding the reductions in hs-CRP levels and HR and preservation of MBP. The effects of DMT were additive. Thus, early donepezil treatment, either monotherapy or in combination with metoprolol, may be a novel pharmacotherapy, as an adjunct therapy to $\mathrm{PCl}$, to improve the long-term prognosis in patients with $\mathrm{AMI}$.

\section{Limitations}


In this study, we used an RMI-induced CHF rat model to evaluate the precarious clinical condition of hospitalised patients with AMI. However, the experimental animals were young, with possible preservation of autonomic function, and were more responsive to various therapeutic interventions. However, many patients with AMI are middle- to old-aged and may have limited capacity for response to the testing of this novel treatment. Furthermore, because clinical trials usually involve patients with various backgrounds of pharmacological treatment, it is difficult to determine the efficacy of monotherapy in that population. This may be an important factor to be considered when translating the outcomes of this basic study to clinical practice.

\section{Conclusions}

Donepezil monotherapy or in combination with metoprolol significantly improved myocardial salvage, reduced $\mathrm{MI}$ size, limited the progression of cardiac remodeling and dysfunction, and prevented subsequent CHF. This suggests that donepezil, with or without metoprolol, may be a potential candidate for post-RMI therapy.

\section{Declarations}

\section{Funding}

This work was partly supported by JSPS KAKENHI (grant numbers 17K09544, 18K08091)

\section{Conflicts of interest/Competing interests}

None declared.

\section{Ethics approval}

The care of animals and all animal experiments were performed in strict accordance with the guiding principles of the Physiological Society of Japan and the Guide for the Care and Use of Laboratory Animals published by the US National Institutes of Health (NIH Publications No 85-23, revised 1996). All protocols were reviewed and approved by the Animal Subject Committee in the National Cerebral and Cardiovascular Center.

\section{Consent to participate}

Not applicable

\section{Consent for publication}

Not applicable

\section{Availability of data and material}


The datasets used and/or analyzed during the current study are available from the corresponding author on reasonable request.

\section{Code availability}

None

\section{Author contributions}

$M L$ and $C Z$ designed the study. $M L$ and $C Z$ performed the measurements and statistical analyses and drafted the manuscript. TK, KU, MI, TN, KS and MS joined in interpreting the data. ML and CZ wrote and edited, TK and KS reviewed the manuscript. All authors have read and approved the final manuscript.

\section{References}

1. Ozaki Y, Katagiri Y, Onuma Y, Amano T, Muramatsu T, Kozuma K, et al. CVIT expert consensus document on primary percutaneous coronary intervention (PCI) for acute myocardial infarction (AMI) in 2018. Cardiovasc Interv Ther. 2018;33(2):178-203. doi:10.1007/s12928-018-0516-y.

2. Velagaleti RS, Pencina MJ, Murabito JM, Wang TJ, Parikh NI, D'Agostino RB, et al. Long-term trends in the incidence of heart failure after myocardial infarction. Circulation. 2008;118(20):2057-62. doi:10.1161/CIRCULATIONAHA.108.784215.

3. Gagnor A, Tomassini F, Romagnoli E, Infantino V, Rosa Brusin MC, Maria C, et al. Percutaneous left main coronary disease treatment without on-site surgery back-up in patients with acute coronary syndromes: immediate and 2-year outcomes. Catheter Cardiovasc Interv. 2012;79(6):979-87. doi:10.1002/ccd.23225.

4. Yellon DM, Hausenloy DJ. Myocardial reperfusion injury. N Engl J Med. 2007;357(11):1121-35. doi:10.1056/NEJMra071667.

5. Ibanez B, James S, Agewall S, Antunes MJ, Bucciarelli-Ducci C, Bueno H, et al. 2017 ESC Guidelines for the management of acute myocardial infarction in patients presenting with ST-segment elevation: The Task Force for the management of acute myocardial infarction in patients presenting with STsegment elevation of the European Society of Cardiology (ESC). Eur Heart J. 2018;39(2):119-77. doi:10.1093/eurheartj/ehx393.

6. Ibanez B, Cimmino G, Prat-Gonzalez S, Vilahur G, Hutter R, Garcia MJ, et al. The cardioprotection granted by metoprolol is restricted to its administration prior to coronary reperfusion. Int $\mathrm{J}$ Cardiol. 2011;147(3):428-32. doi:10.1016/j.ijcard.2009.09.551.

7. Ibanez B, Prat-Gonzalez S, Speidl WS, Vilahur G, Pinero A, Cimmino G, et al. Early metoprolol administration before coronary reperfusion results in increased myocardial salvage: analysis of ischemic myocardium at risk using cardiac magnetic resonance. Circulation. 2007;115(23):2909-16. doi:10.1161/CIRCULATIONAHA.106.679639. 
8. Cimmino G, Ibanez B, Giannarelli C, Prat-Gonzalez S, Hutter R, Garcia M, et al. Carvedilol administration in acute myocardial infarction results in stronger inhibition of early markers of left ventricular remodeling than metoprolol. Int J Cardiol. 2011;153(3):256-61. doi:10.1016/j.ijcard.2010.08.018.

9. Ibanez B, Macaya C, Sánchez-Brunete V, Pizarro G, Fernández-Friera L, Mateos A, et al. Effect of early metoprolol on infarct size in ST-segment-elevation myocardial infarction patients undergoing primary percutaneous coronary intervention: the Effect of Metoprolol in Cardioprotection During an Acute Myocardial Infarction (METOCARD-CNIC) trial. Circulation. 2013;128(14):1495-503. doi:10.1161/CIRCULATIONAHA.113.003653.

10. Roolvink V, Ibanez B, Ottervanger JP, Pizarro G, van Royen N, Mateos A, et al. Early Intravenous BetaBlockers in Patients With ST-Segment Elevation Myocardial Infarction Before Primary Percutaneous Coronary Intervention. J Am Coll Cardiol. 2016;67(23):2705-15. doi:10.1016/j.jacc.2016.03.522.

11. Floras JS. Clinical aspects of sympathetic activation and parasympathetic withdrawal in heart failure. J Am Coll Cardiol. 1993;22(4 Suppl A):72A-84A.

12. La Rovere MT, Bigger JT, Jr., Marcus FI, Mortara A, Schwartz PJ. Baroreflex sensitivity and heart-rate variability in prediction of total cardiac mortality after myocardial infarction. ATRAMI (Autonomic Tone and Reflexes After Myocardial Infarction) Investigators. Lancet. 1998;351(9101):478-84.

13. Li M, Zheng C, Sato T, Kawada T, Sugimachi M, Sunagawa K. Vagal nerve stimulation markedly improves long-term survival after chronic heart failure in rats. Circulation. 2004;109(1):120-4.

14. Uemura K, Zheng C, Li M, Kawada T, Sugimachi M. Early short-term vagal nerve stimulation attenuates cardiac remodeling after reperfused myocardial infarction. J Card Fail. 2010;16(8):68999. doi:10.1016/j.cardfail.2010.03.001.

15. Li M, Zheng C, Kawada T, Inagaki M, Uemura K, Shishido T, et al. Donepezil markedly improves longterm survival in rats with chronic heart failure after extensive myocardial infarction. Circ $\mathrm{J}$. 2013;77(10):2519-25.

16. Li M, Zheng C, Kawada T, Inagaki M, Uemura K, Sugimachi M. Adding the acetylcholinesterase inhibitor, donepezil, to losartan treatment markedly improves long-term survival in rats with chronic heart failure. Eur J Heart Fail. 2014;16(10):1056-65. doi:10.1002/ejhf.164.

17. Li M, Zheng C, Kawada T, Inagaki M, Uemura K, Sugimachi M. Chronic vagal nerve stimulation exerts additional beneficial effects on the beta-blocker-treated failing heart. J Physiol Sci. 2019;69(2):295303. doi:10.1007/s12576-018-0646-0.

18. Couto GD, Liu W, Tseliou E, Sun B, Makkar N, Kanazawa H, et al. Macrophages mediate cardioprotective cellular postconditioning in acute myocardial infarction. $\mathrm{J}$ Clin Invest. 2015;125(8):3147-62. doi:10.1172/JCl81321.

19. Li Y, Xie Z, Wang Y, Hu H. Macrophage M1/M2 polarization in patients with pregnancy-induced hypertension. Can J Physiol Pharmacol. 2018;96(9):922-8. doi:10.1139/cjpp-2017-0694.

20. Wu E, Ortiz JT, Tejedor P, Lee DC, Bucciarelli-Ducci C, Kansal P, et al. Infarct size by contrast enhanced cardiac magnetic resonance is a stronger predictor of outcomes than left ventricular ejection fraction 
or end-systolic volume index: prospective cohort study. Heart. 2008;94(6):730-6. doi:10.1136/hrt.2007.122622.

21. Li M, Zheng C, Kawada T, Inagaki M, Uemura K, Akiyama T, et al. Impact of Peripheral a7-Nicotinic Acetylcholine Receptors on Cardioprotective Effects of Donepezil in Chronic Heart Failure Rats. Cardiovasc Drugs Ther. 2020. doi:10.1007/s10557-020-07062-1.

22. Li M, Zheng C, Kawada T, Inagaki M, Uemura K, Sugimachi M. Intracerebroventricular infusion of donepezil prevents cardiac remodeling and improves the prognosis of chronic heart failure rats. $J$ Physiol Sci. 2020;70(1):11. doi:10.1186/s12576-020-00739-0.

23. Kawada T, Li M, Kamiya A, Shimizu S, Uemura K, Yamamoto H, et al. Open-loop dynamic and static characteristics of the carotid sinus baroreflex in rats with chronic heart failure after myocardial infarction. J Physiol Sci. 2010;60(4):283-98. doi:10.1007/s12576-010-0096-9.

24. Suga H. Ventricular energetics. Physiol Rev. 1990;70(2):247-77. doi:10.1152/physrev.1990.70.2.247.

25. Kawada T, Yamazaki T, Akiyama T, Li M, Ariumi H, Mori H, et al. Vagal stimulation suppresses ischemia-induced myocardial interstitial norepinephrine release. Life Sci. 2006;78(8):882-7. doi:10.1016/j.lfs.2005.05.087.

26. Kapur NK, Alkhouli MA, DeMartini TJ, Faraz H, George ZH, Goodwin MJ, et al. Unloading the Left Ventricle Before Reperfusion in Patients With Anterior ST-Segment-Elevation Myocardial Infarction. Circulation. 2019;139(3):337-46. doi:10.1161/CIRCULATIONAHA.118.038269.

27. Menees DS, Peterson ED, Wang Y, Curtis JP, Messenger JC, Rumsfeld JS, et al. Door-to-balloon time and mortality among patients undergoing primary PCI. N Engl J Med. 2013;369(10):901-9. doi:10.1056/NEJMoa1208200.

28. Stone GW, Selker HP, Thiele H, Patel MR, Udelson JE, Ohman EM, et al. Relationship Between Infarct Size and Outcomes Following Primary PCl: Patient-Level Analysis From 10 Randomized Trials. J Am Coll Cardiol. 2016;67(14):1674-83. doi:10.1016/j.jacc.2016.01.069.

\section{Figures}




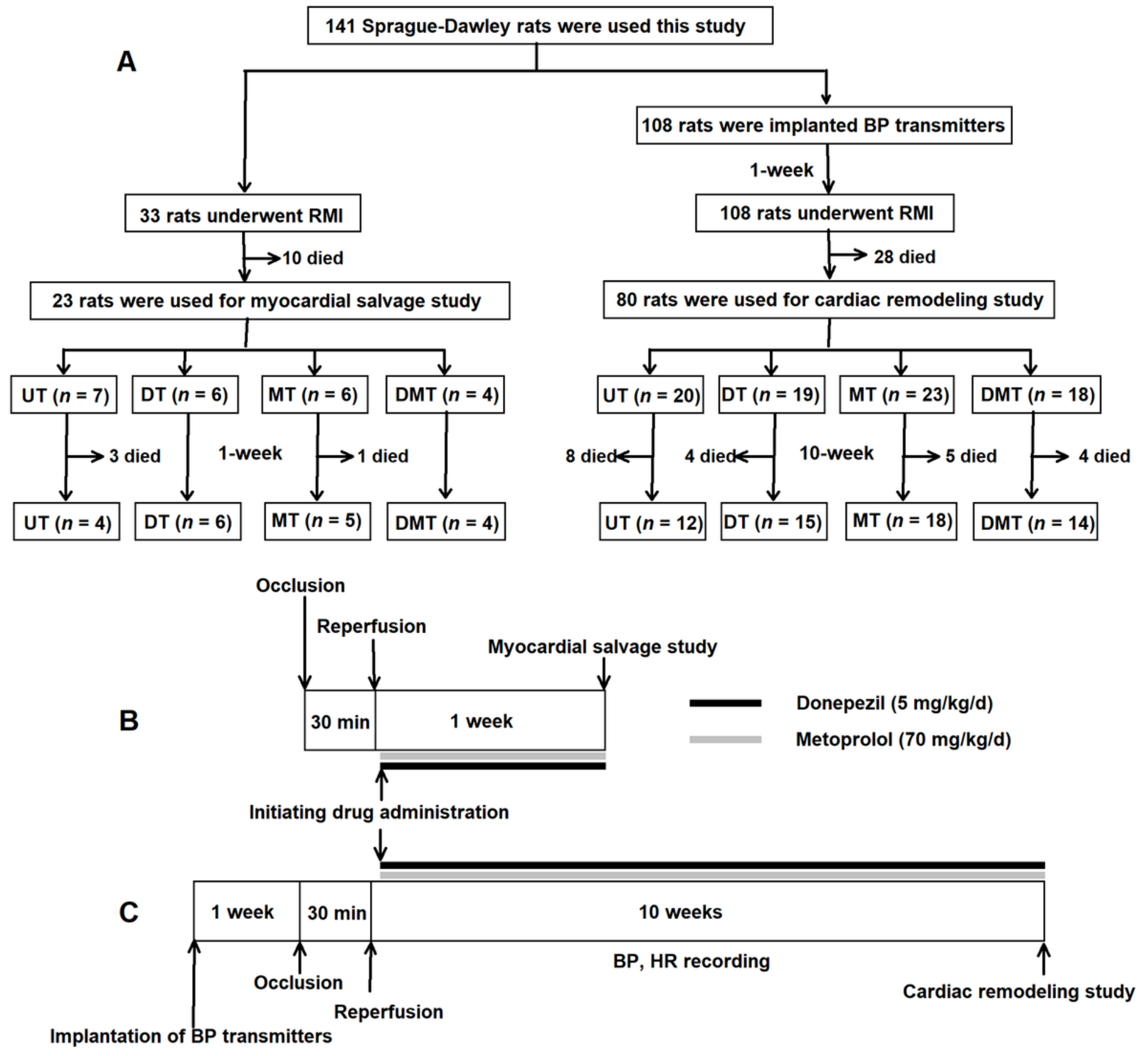

Figure 1

Experimental design and timeline. (A) Experimental design of donepezil therapy in rats with chronic heart failure with reperfused myocardial infarction (RMI). (B, C) Experimental events and timelines. UT, untreated; DT, donepezil treatment; MT, metoprolol treatment; DMT, donepezil plus metoprolol treatment. 

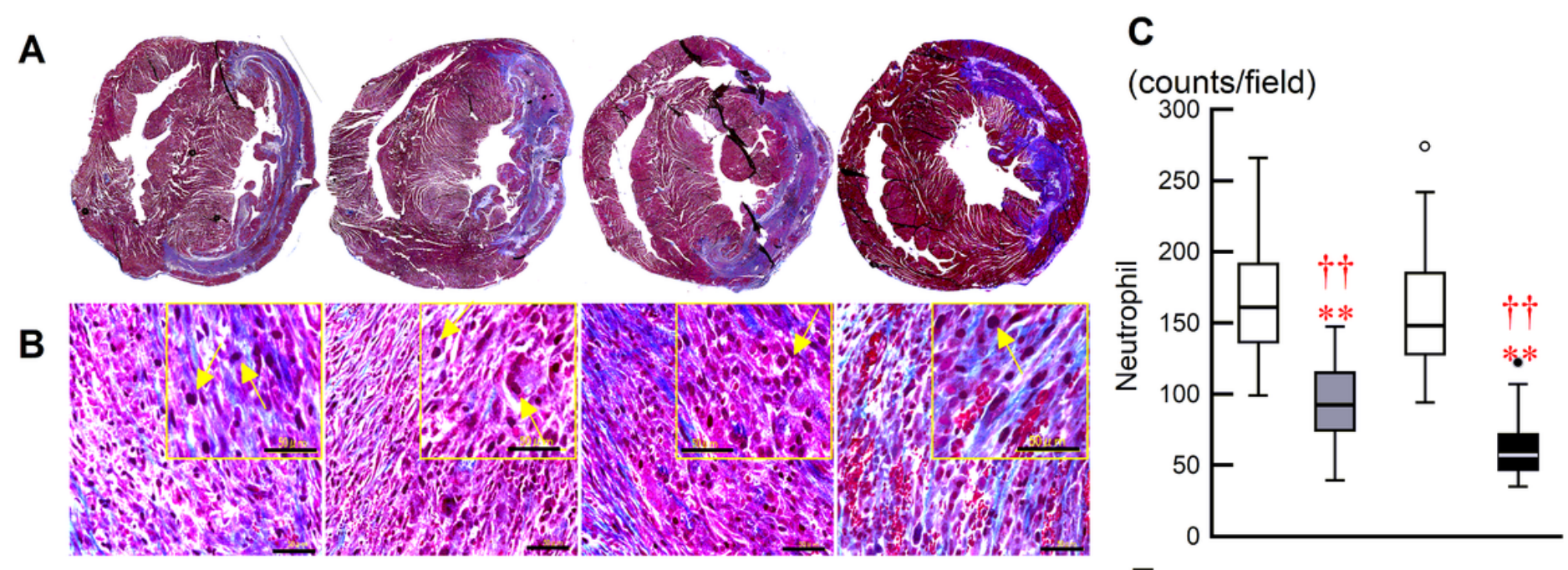

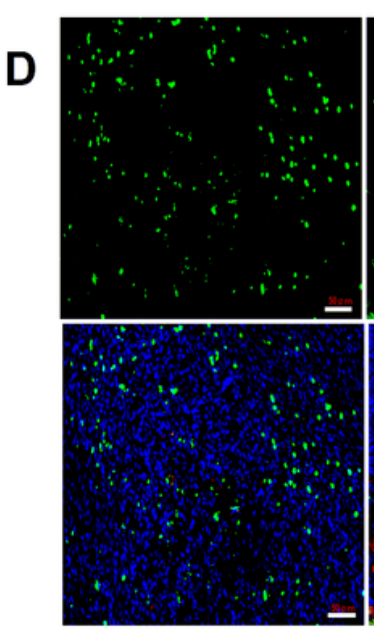

UT

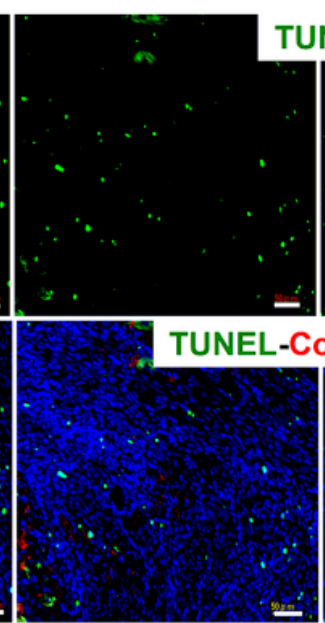

DT

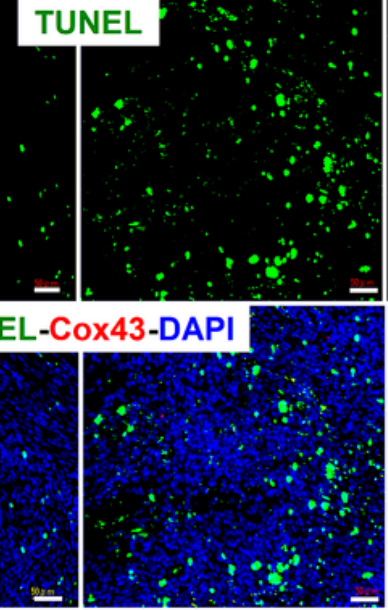

MT

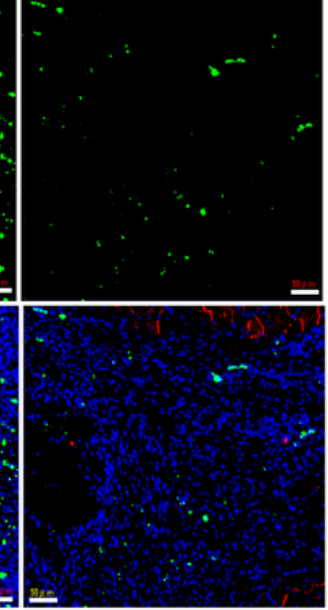

DMT
$E$

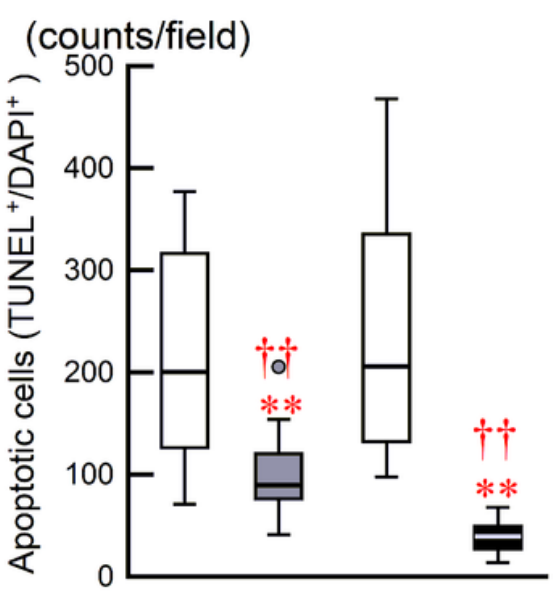

UT DT MT DMT

\section{Figure 2}

Masson's trichrome staining and immunohistochemical analyses on day 8 after reperfused myocardial infarction (RMI). Representative photomicrographs of Masson's trichrome staining of the heart $(A)$ and the ischaemic/reperfusion (I/R) region (B). In (B), arrows indicate infiltrating neutrophils. Scale bar, 50 $\mu \mathrm{m}$. (C) Neutrophil counts in the I/R region (UT: $166 \pm 8 /$ field, $n=4,24$ fields; DT: $93 \pm 6 /$ field, $n=4,24$ fields; MT: $157 \pm$ 10/field, $n=4,24$ fields; DMT: $63 \pm 5 /$ field, $n=4,24$ fields). (D) Representative micrographs of TUNEL, connexin 43, and DAPI staining of the I/R region. (E) Quantitative analysis of apoptotic cells (UT: $213 \pm 3 /$ field, $n=4,24$ fields; DT: $99 \pm 8 /$ field, $n=6,36$ fields; MT: $236 \pm 31 /$ field, $n=$ 5, 30 fields; DMT: $39 \pm 2 /$ field, $n=4,24$ fields). Data are expressed as means \pm SEMs. (C, E) Box-andwhisker plots based on neutrophil counts and apoptotic cells in all fields of each group. **P < 0.01 vs. UT, ††P $<0.01$ vs.MT, using nonparametric Kruskal-Wallis tests, followed by Dunn's test for all pairwise comparisons. UT, untreated; DT, donepezil treatment; MT, metoprolol treatment; DMT, donepezil plus metoprolol treatment. 

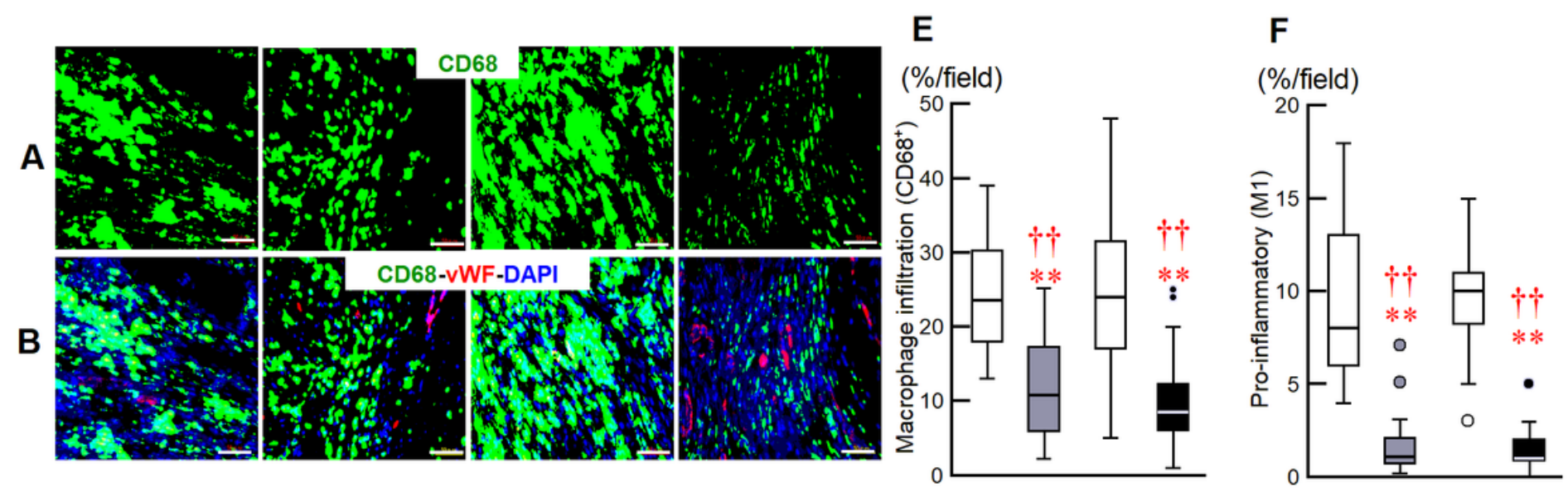

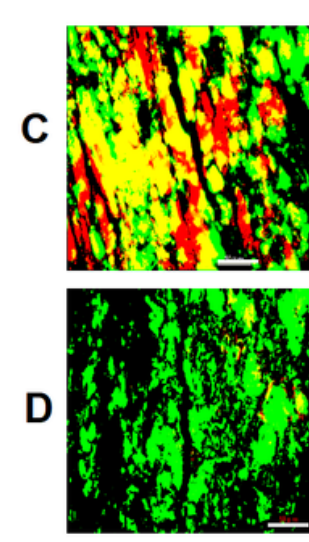

UT
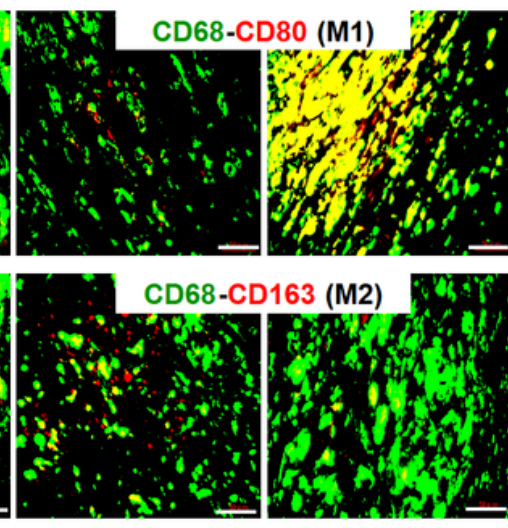

DT

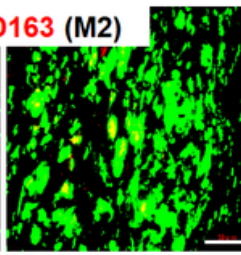

MT

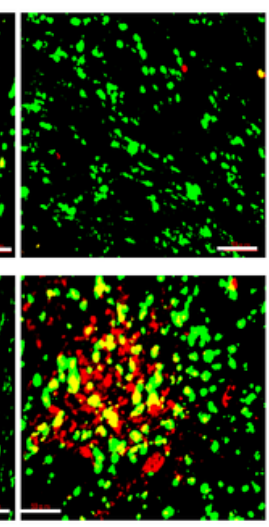

DMT

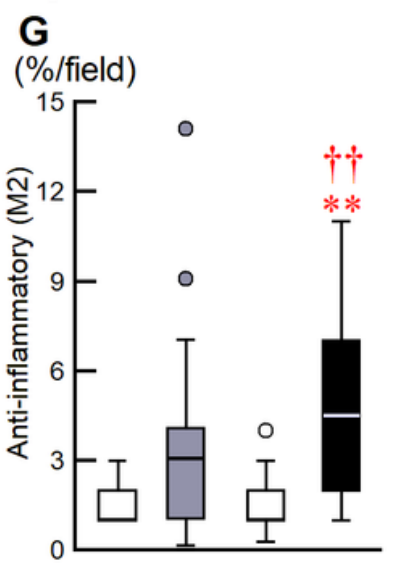

UT DT MT DMT

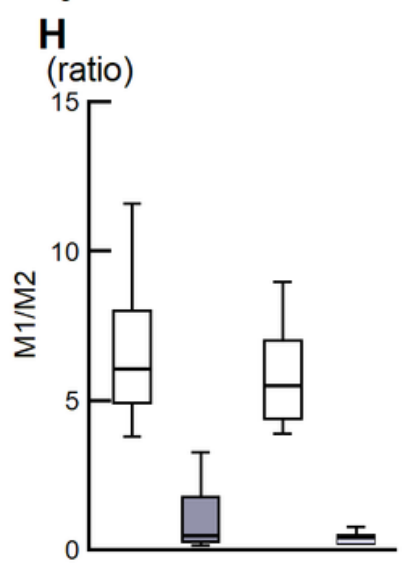

UT DT MT DMT

Figure 3

Analysis of macrophages on day 8 post-RMI. Representative micrographs of CD68 (A), and triple CD68, VWF, and DAPI staining of the I/R region (B). (C) Representative macrographs of CD68 and CD80 cells (pro-inflammatory M1), and CD68 and CD163 cells (anti-inflammatory M2) in the I/R region. Scale bar, 50 $\mu \mathrm{m}$. (E) Quantitative analysis of macrophage infiltration (UT:24 $\pm 3 \% /$ field, $n=4,24$ fields of view; DT: 11 $\pm 2 \% /$ field, $n=6,36$ fields; MT: $24 \pm 2 \% /$ field, $n=5,30$ fields; DMT: $10 \pm 3 \% /$ field, $n=4,32$ fields). (F) M1 infiltration (UT: $9.4 \pm 0.8 \% /$ field, $n=4,24$ fields; DT: $1.6 \pm 0.9 \% /$ field, $n=5,30$ fields; MT: $9.8 \pm 0.5 \% /$ field, $\mathrm{n}=5,30$ fields; DMT: $1.5 \pm 0.2 \% /$ field, $\mathrm{n}=4,24$ fields). (G) M2 infiltration (UT: $1.5 \pm 0.1 \% /$ field, $n=4,24$ fields; DT: $3.1 \pm 0.5 \% /$ field, $n=5,30$ fields; MT: $1.5 \pm 0.2 \% /$ field, $n=5,30$ fields; DMT: $4.8 \pm 0.6 \% /$ field, $n=$ 4, 24 fields). (H) M1 to M2 ratio (UT: $6.9 \pm 1.7, n=4$; DT: $1.1 \pm 0.6, n=5$; MT: $6.0 \pm 0.9, n=5$; DMT: $0.4 \pm$ $0.1, n=4)$. M1: macrophage type $1 ; M 2$ : macrophage type 2. Data are expressed as means \pm SEMs. (EH) Box-and-whisker plots based on macrophage, M1, and M2 infiltration and the M1/M2 ratio in all fields. **P $<0.01$ vs. UT, $++P<0.01$ vs. MT, using nonparametric Kruskal-Wallis tests, followed by Dunn's test for all pairwise comparisons. UT, untreated; DT, donepezil treatment; MT, metoprolol treatment; DMT, donepezil plus metoprolol treatment. 


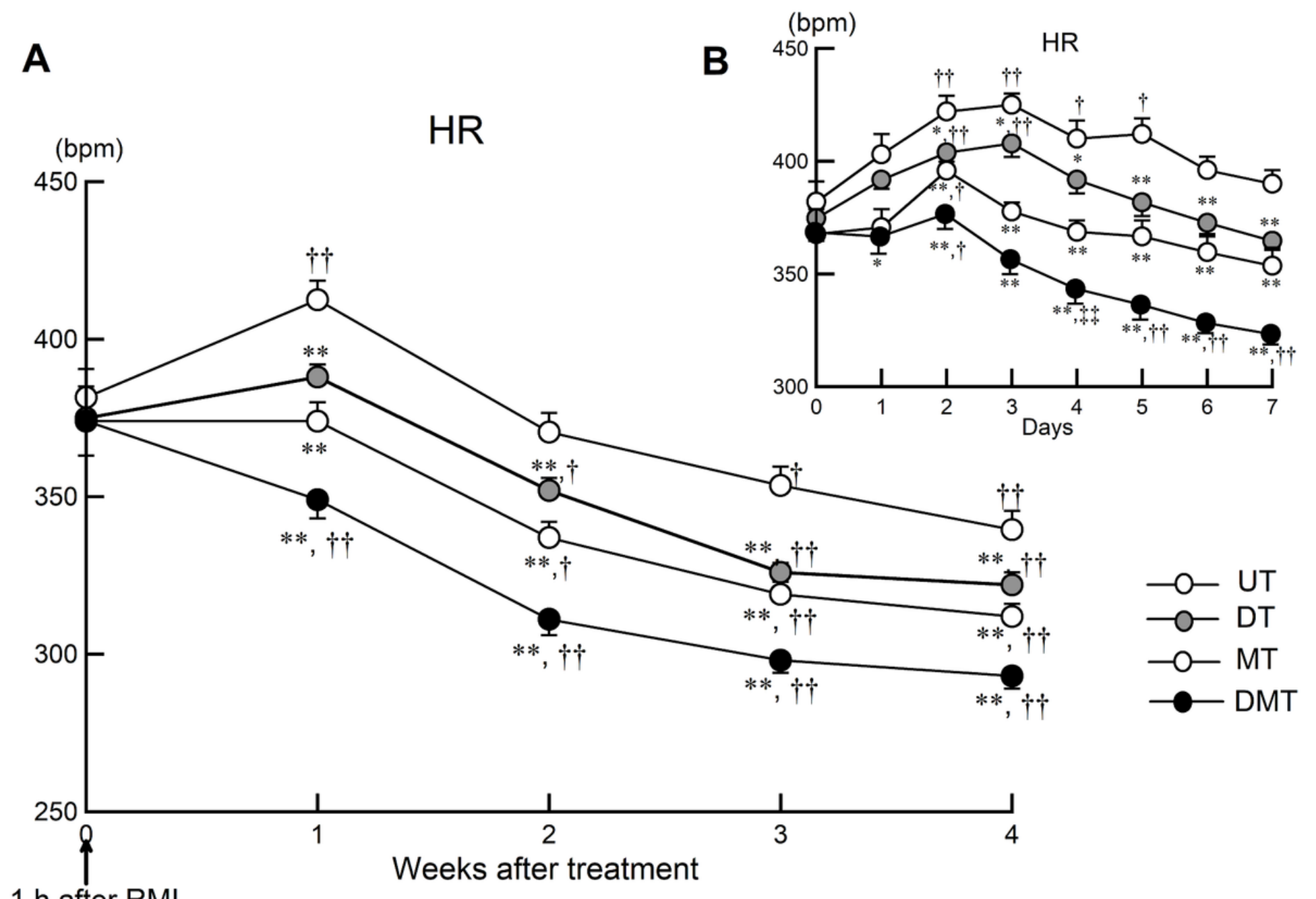

\section{$1 \mathrm{~h}$ after RMI}

Figure 4

Effects of the treatment on heart rate (HR) of rats with RMl-induced CHF, recorded using telemetry. (A) Weekly average HR. Each point represents average 1-week data for all animals in each group. (B) Daily average HR during week 1 . Each point represents the average 1-day data for all animals in each group. (UT, $n=14 ; D T, n=16 ; M T, n=18 ; D M T, n=14$ ). The difference in HR between the DT and UT groups was approximately $20 \mathrm{bpm}$ in week 4 (322 \pm 4 vs. $340 \pm 4 \mathrm{bpm}$, respectively, $\mathrm{P}<0.01$ ); and the difference between the MT and UT groups was approximately $30 \mathrm{bpm}(312 \pm 4$ vs. $340 \pm 4 \mathrm{bpm}$, respectively, $\mathrm{P}<$ 0.01). The difference in HR between the DMT and UT groups was up to $50 \mathrm{bpm}(293 \pm 3 \mathrm{vs.} 340 \pm 4 \mathrm{bpm}$, respectively, $P<0.01)$. Values are expressed as means $\pm S E M s . t P<0.05, t+P<0.01$ vs. pretreatment value (week 0 ) in each group; ${ }^{*} P<0.05,{ }^{*} \mathrm{P}<0.01$ vs. UT group, using repeated measures one-way ANOVA and post-hoc Dunnett's test. UT, untreated; DT, donepezil treatment; MT, metoprolol treatment; DMT, donepezil plus metoprolol treatment. 

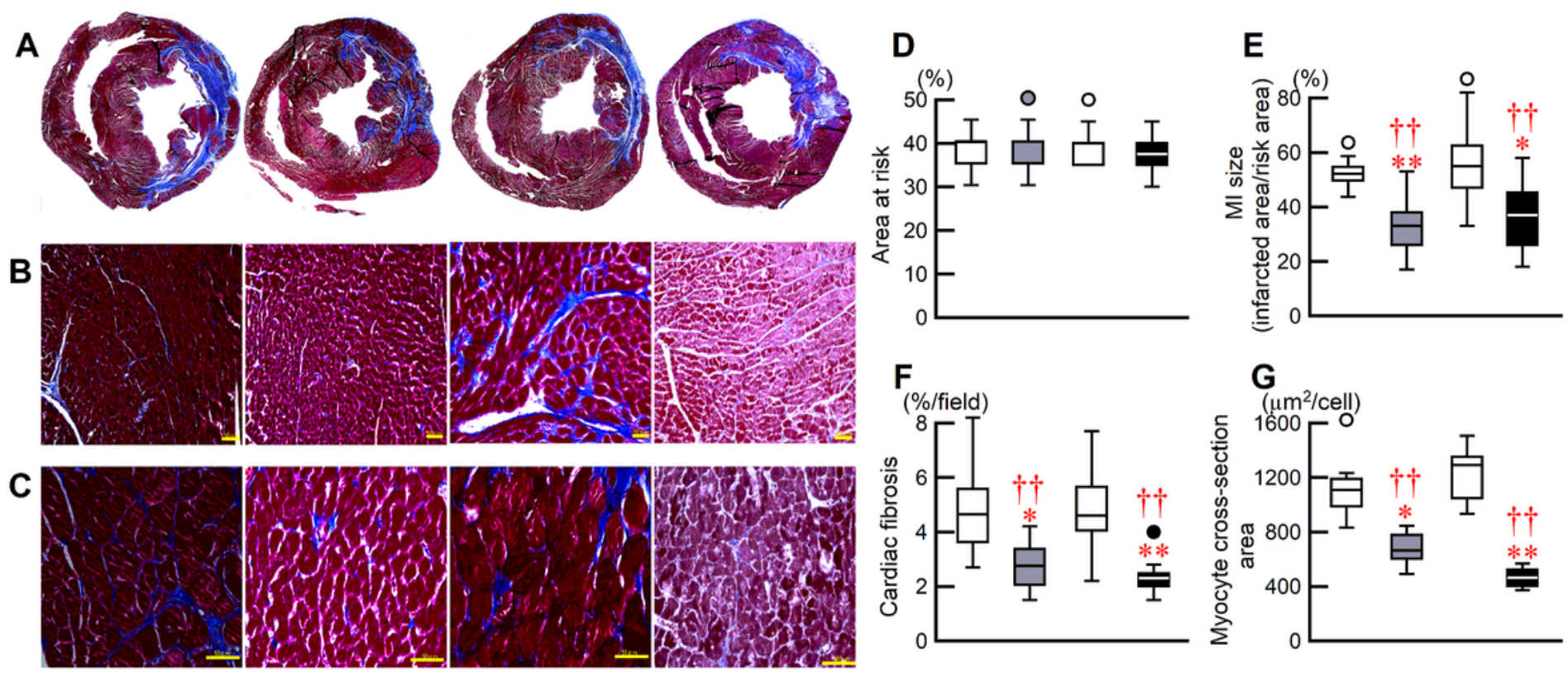

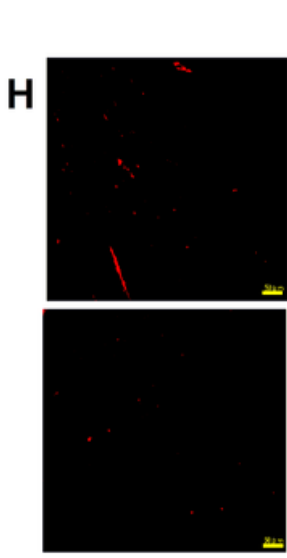

UT
Microvessels (vWF positive)
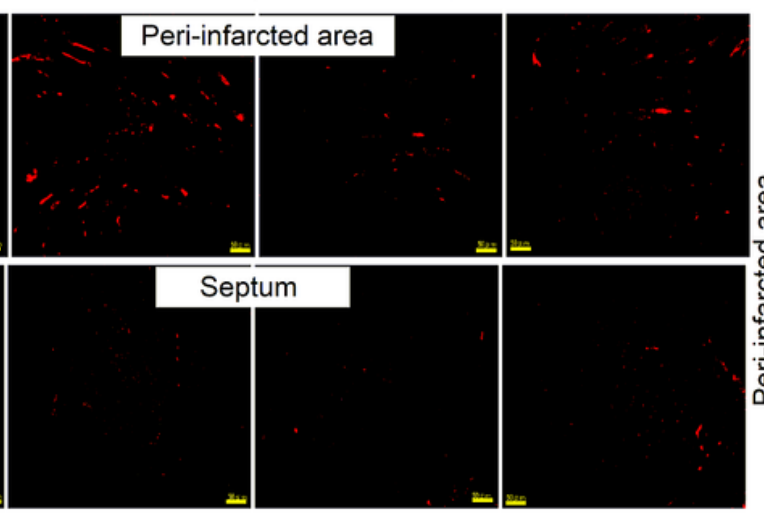

DT

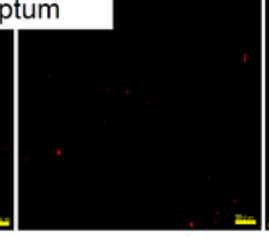

MT

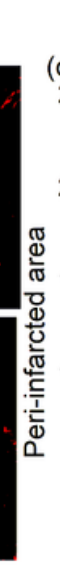

DMT
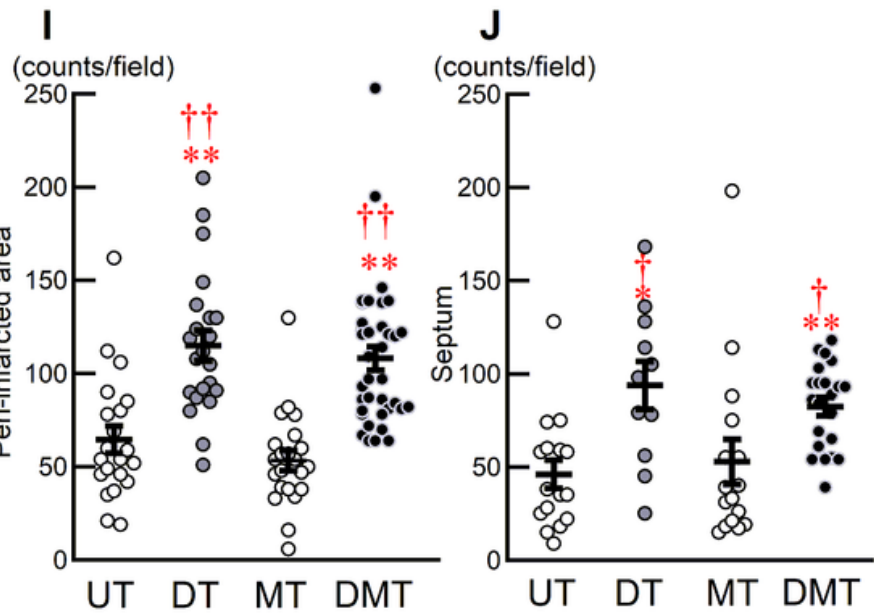

\section{Figure 5}

Masson's trichrome staining of ventricles in rats with CHF 10 weeks after treatment. (A) Representative biventricular sections of RMI. (B) Representative images of cardiac fibrosis in the noninfarcted area. (C) Representative myocyte cross-sections. Scale bar, $50 \mu \mathrm{m}$. (D) Area at risk (UT, $38 \pm 5 \%, n=12 ; \mathrm{DT}, 38 \pm$ $6 \%, n=15 ; M T, 38 \pm 4 \%, n=18 ; D M T, 39 \pm 5 \%, n=14$ ). (E) Infarct size (infarcted area/area at risk) (UT, 51 $\pm 2 \%, n=12 ;$ DT, $33 \pm 3 \%, n=15 ; M T, 56 \pm 3 \%, n=18 ; D M T, 35 \pm 3 \%, n=14)$. (F) Cardiac fibrosis index in the non-infarcted regions (UT, $5.0 \pm 0.6 \%$ /field, $n=10,60$ fields of view; DT, $2.7 \pm 0.2 \% /$ field, $n=14,84$ fields; MT, $4.9 \pm$ 0.3\%/field, $n=18,108$ fields; DMT, $2.4 \pm 0.2 \% /$ field, $n=14,84$ fields). (G) Myocyte crosssectional area in the noninfarcted region (UT, $1118 \pm 98 \mu \mathrm{m} 2 /$ cell, $n=7,312$ cells; DT, $683 \pm 34 \mu \mathrm{m} 2 /$ cell, $\mathrm{n}=12,668$ cells; MT, $1218 \pm 123 \mu \mathrm{m} 2 /$ cell, $\mathrm{n}=7,264$ cells; DMT, $465 \pm 33 \mu \mathrm{m} 2 /$ cell, $\mathrm{n}=6,869$ cells). (H) Representative micrographs of blood microvessel (von Willebrand factor [vWF, red]) immunostaining in the peri-infarct and noninfarcted (septum) regions in rats with CHF. Scale bar, $50 \mu \mathrm{m}$. Quantitative analysis of microvessel density in (I) peri-infarct region (UT, $64 \pm 10 /$ field, $n=6,21$ fields of view; DT, 119 \pm 11/field, $n$ = 6, 21 fields; MT, $52 \pm$ 7/field, $n$ = 6, 22 fields; DMT, $103 \pm$ 8/field, $n$ = 7, 36 fields); and (J) in 
the septum (UT, $44 \pm 8 /$ field, $n=6,16$ fields; DT, $105 \pm 18$ / field, $n=6,11$ fields; MT, $62 \pm 20 /$ field, $n=6,16$ fields; DMT, $82 \pm 8 /$ field, $n=7,22$ fields). Values are expressed as means \pm SEMs. Data are shown as box-and-whisker plots based on area at risk, Ml size (infarcted area / area at risk), cardiac fibrosis, and myocyte cross-section area in in all fields. ${ }^{P}<0.05,{ }^{*} \mathrm{P}<0.01 \mathrm{vs}$. UT; $\mathrm{tP}<0.05, \mathrm{t} \mathrm{P}<0.01$ vs. MT, using nonparametric Kruskal-Wallis tests, followed by Dunn's tests for all pairwise comparisons. UT, untreated; DT, donepezil treatment; MT, metoprolol treatment; DMT, donepezil plus metoprolol treatment.

\section{Supplementary Files}

This is a list of supplementary files associated with this preprint. Click to download.

- Supplementarymateriallisubmission.doc 